\title{
Analysis and Design of Class E Frequency Multiplier With Shunt Linear and Nonlinear Capacitances at Any Duty Cycle
}

This paper was downloaded from TechRxiv (https://www.techrxiv.org).

\section{LICENSE}

CC BY 4.0

SUBMISSION DATE / POSTED DATE

25-08-2021 / 27-08-2021

\section{CITATION}

yao, jinbo (2021): Analysis and Design of Class E Frequency Multiplier With Shunt Linear and Nonlinear Capacitances at Any Duty Cycle. TechRxiv. Preprint. https://doi.org/10.36227/techrxiv.16436178.v1

$\mathrm{DOI}$

10.36227/techrxiv.16436178.v1 


\title{
Analysis and Design of Class E Frequency Multiplier With Shunt Linear and Nonlinear Capacitances at Any Duty Cycle
}

\author{
Jinbo Yao, Mingyu Li, Member, IEEE, Xuefeng Yu, Tian Li, and Zhijiang Dai, Member, IEEE,
}

\begin{abstract}
The theoretical efficiency of the class $\mathbf{E}$ frequency multiplier with arbitrary output frequency can reach $100 \%$ by controlling the phase of the output current to satisfy the ZVS/ZVDS condition. It is necessary to select the appropriate value of the shunt capacitance of the frequency multiplier for satisfying these two conditions. In general, the shunt capacitance of the transistor consists of the linear external capacitance and the nonlinear parasitic capacitance. Therefore, the conventional design method of treating shunt capacitance as purely linear is not accurate enough. In this paper, a class $E$ frequency multiplier with shunt linear and nonlinear capacitances at any duty cycle is analyzed and designed, and the design curve is given in detail. The simulation and experimental results shows highly agreement with the theoretical analysis result.
\end{abstract}

Index Terms-Class E, frequency multiplier, zero-voltage switching (ZVS), zero-voltage-deravate switching(ZVDS)

\section{INTRODUCTION}

$\mathbf{T}$ HE class-E power amplifiers (PAs) have been concerned by the academic community for their simple circuits, and the extremely higher efficiency can be obtained when the zero-voltage switching and zero-voltage derivative switching (ZVS/ZVDS) conditions are satisfied [1]-[4]. The Class E frequency multiplier, which only changes the resonant frequency of the series resonant circuit on the Class E circuit, also has the above two characteristics and can be used as the driver circuit [5]. And the mathematical derivation and design equation for the frequency multiplier are analyzed and given in [6]-[8]. These papers indicate that the duty cycle of the frequency multiplier must be less than $1 / \mathrm{n}$ ( $\mathrm{n}$ refers to the multiple of the frequency multiplier output frequency to the drive signal), otherwise the drain-source voltage will be negative and is harmful to the circuit. Furthermore, the influence of duty cycle on the performance of frequency multiplier is analyzed in [9] and design equations under different $Q$ and $\mathrm{n}$ values are given in detail. Meanwhile, two different topologies to enable the frequency multiplier to work with duty cycle of 0.5 are proposed in [10]-[11]. However, the shunt capacitors which are composed of linear capacitors and nonlinear capacitors are not take into account in these papers.

Manuscript received xx, 2021; revised xx, 2021; accepted xx, 2021.(Corresponding Auther: Mingyu Li)

This work was supported by the National Natural Science Foundation of China under 62171068

Jinbo Yao, Mingyu Li, Tian Li, and Zhijiang Dai are with School of Microelectronics and Communication Engineering, Chongqing University, Chongqing 400044, China(e-mail: myli@cqu.edu.cn). Xuefeng Yu is with the Xi' an branch of China Academy of Space Technology, Xi' an 710100, China.
In fact, the shunt capacitance value is vital for achieving the optimum conditions for the class E circuit. Some papers have been pointed out that the linearity and nonlinearity of the shunt capacitance have different effects on the performance of the class E circuit [12]-[17]. For example, the case that the shunt capacitance is composed of linear capacitance and nonlinear capacitance is considered for the class E amplifier design in [16], and the design equation through numerical solution is given only when the duty cycle is 0.5 . Furthermore, the design of the class $\mathrm{E}$ amplifier with the shunt linear and nonlinear capacitances under arbitrary duty cycle is proposed in [17]. In the practical application of the frequency multiplier circuit, the inherent nonlinear parasitic capacitance of the MOSFET is difficult to be exactly applicable for the design specification. And a suitable external capacitor is usually required to make the total shunt capacitance conforming to the design specification. Therefore, the effects of the shunt linear and nonlinear capacitances on the performance of the frequency multiplier circuit must be taken into account.

In this paper, the analytical derivation of the class $\mathrm{E}$ frequency multipliers with shunt linear and nonlinear capacitance, which satisfies the ZVS/ZVDS conditions at any duty ratio, is given. The mathematical relationship of the circuit parameters as the functions of the duty ratio are derived in detail. A frequency doubler circuit example is designed for verifying the proposed design method. The simulation and the experimental results show the usefulness and effectiveness of the analytical design approach for the class $\mathrm{E}$ frequency multipliers at any duty ratio.

\section{Circuit AnAlysis}

A basic circuit of class $\mathrm{E}$ frequency multiplier is shown in Fig.1. In this circuit, $C_{o}$ is the device'sdevice's parasitic nonlinear capacitance, and $C_{e}$ is the external linear capacitance, $C_{o}$ and $C_{e}$ make up the shunt capacitance of the frequency multiplier circuit together. The analysis of the class E frequency multiplier of Fig.1 is carried out under the following assumptions.

1) The inductance of the choke coil $L_{R F C}$ is high enough that the current $I_{D D}$ flowing through it could be considered constant.

2) The loaded quality factor $Q$ of the circuit is large enough that the output current waveform is pure sinusoidal.

3) All component resistances and switch-on resistances are ignored, so that the input power is equal to the output power if the soft switch condition is satisfied. 


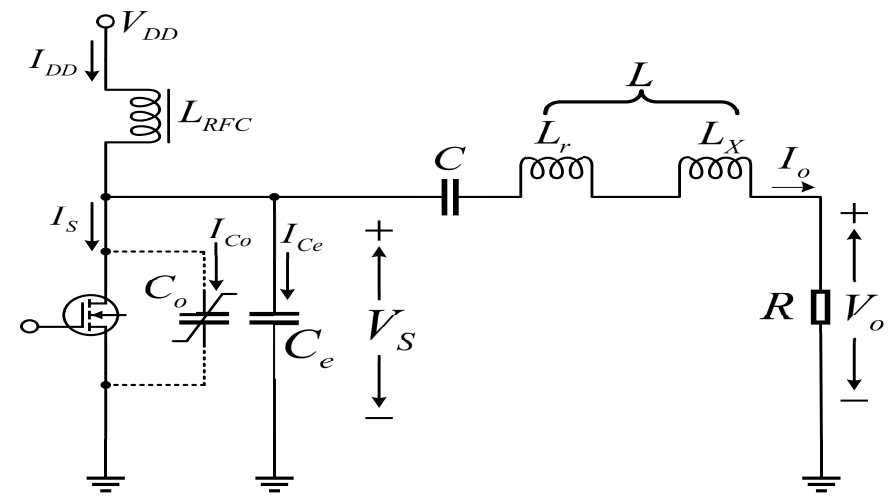

Fig. 1. Basic circuit of class E multiplier

4) The device switch turns on and turns off so quick, that it can be considered instantaneous and no power loss.

5) The duty cycle is arbitrary under the basic limits of the frequency multiplier.

6) The total shunt capacitance $C_{d s}$ is equal to the nonlinear capacitance $C_{o}$ plus the linear capacitance $C_{e}$, which is described by

$$
C_{d s}=C_{o}+C_{e}=\frac{C_{j 0}}{\left(1+\frac{V_{S}}{V_{b i}}\right)^{m}}+C_{e}
$$

where $C_{j 0}$ is the output capacitance of the device when the drain-to-source voltage $V_{s}=0, V_{b i}$ is the built-in potential of the MOSFET body diode, the grading coefficient $m$ is a parameter determined by the device, which is defined as $m=0.5$ in this paper. The nonlinearity of the shunt capacitance is incorporated into the analysis through this equation.

Defining the state of the switch as off when $0<\theta \leq 2 \pi D$ (D is the duty cycle), so no current flows through the switch, i.e. $I_{S}=0$, in this period, applying the KCL law, the current on the overall shunt capacitance is expressed as

$$
I_{C}(\theta)=I_{D D}-I_{n} \sin (n \theta+\Phi)
$$

where $I_{D D}$ is the dc input current, $I_{n}$ is the amplitude of the $n$th harmonic output current, $\Phi$ is the phase shift of the output current and $\theta=\omega t$. Considering the voltage and current relationship across the capacitance, the current through the shunt capacitance could be redefined as

$$
I_{C}(\theta)=I_{C_{o}}(\theta)+I_{C_{e}}(\theta)=\omega\left(C_{o}+C_{e}\right) \frac{d V_{S}}{d \theta}
$$

where $I_{C_{o}}(\theta)$ represents the current flowing through the nonlinear capacitance $C_{o}, I_{C_{e}}(\theta)$ represents the current flowing through the linear capacitance $C_{e}, V_{S}$ represents the voltage across the shunt capacitance. integrating both sides of this equation with respect of $\theta$, the following expressions can be given

$$
\int I_{C}(\theta) d \theta=\omega \int\left(\frac{C_{j 0}}{\sqrt{1+\frac{V_{S}}{V_{b i}}}}+C_{e}\right) d V_{S}
$$

Then substituting (2) into (4), and expanding the integral of both sides, a quadratic equation of $V_{S}$ is yielded, There are two

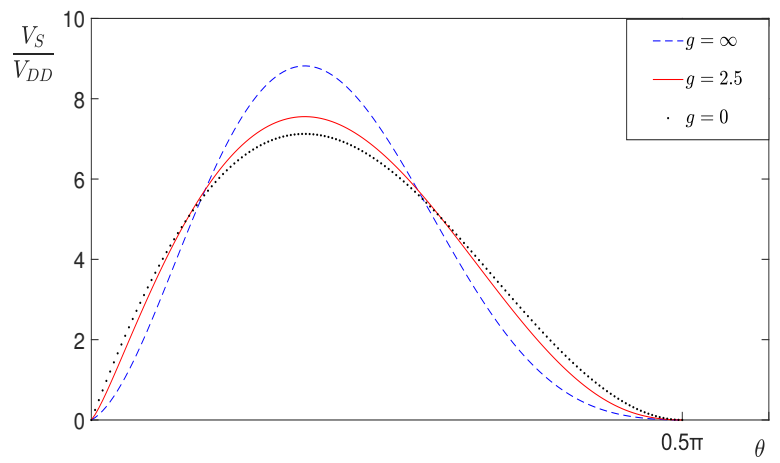

Fig. 2. Waveforms of the switch voltage for $g=\infty, g=2.5$ and $g=0$ at $V_{D D} / V_{b i}=14.29, D=0.25, n=2$.

solutions for this equation, and one of the solutions can not satisfy the ZVS condition, which is dropped. Another solution can satisfy the ZVS condition, which is given as

$$
V_{S}=2 g V_{b i}\left(g+h(\theta)-\sqrt{g^{2}+2 g h(\theta)+1}\right)
$$

where

$$
\begin{gathered}
h(\theta)=\frac{I_{D D} \theta+\frac{I_{n}}{n}(\cos (n \theta+\Phi)-\cos (\Phi))}{2 \omega C_{j 0} V_{b i}}+1 \\
g=\frac{C_{j 0}}{C_{e}}
\end{gathered}
$$

where $h(\theta)$ is an intermediate form for simplifying the equation. And $g$ is the ratio of $C_{j 0}$ to $C_{e}$, which represents the degree of nonlinearity in the shunt capacitance. Fig.2 shows the effect of shunt capacitance on the $V_{S}$ waveform of a frequency doubler at $D=0.25$ in three typical cases, which satisfies the optimum conditions. The three cases are: $g=\infty, g=2.5$ and $g=0$ at $V_{D D} / V_{b i}=14.29$. The derivation of $V_{S}$ at $g=\infty$ is obtained from [12], the waveform at $g=2.5$ is obtained from (5), the waveform at $g=0$ is obtained in [7]. Here $g=\infty$ implies that the shunt capacitance entirely consists of nonlinear capacitance, and the peak voltage in this case is significantly higher than the other two cases. And $g=2.5$ implies the shunt capacitance consists of both nonlinear and linear capacitance, which represents the most common situation in practical applications. The peak voltage in this case is between the other two cases. Finally, $g=0$ implies the shunt capacitance only consist of linear capacitance. The peak voltage is the lowest in three cases.

\section{Circuit Parameters For Optimum Operation at ANY DUTY RATIO}

\section{A. ZVS Condition}

Applying the ZVS condition, that is

$$
V_{S}(2 \pi D)=0
$$

Substituting (8) into (5)

$h(2 \pi D)=\frac{2 \pi D I_{D D}+\frac{I_{n}}{n}(\cos (2 n \pi D+\Phi)-\cos (\Phi))}{2 \omega C_{j 0} V_{b i}}+1=1$ 


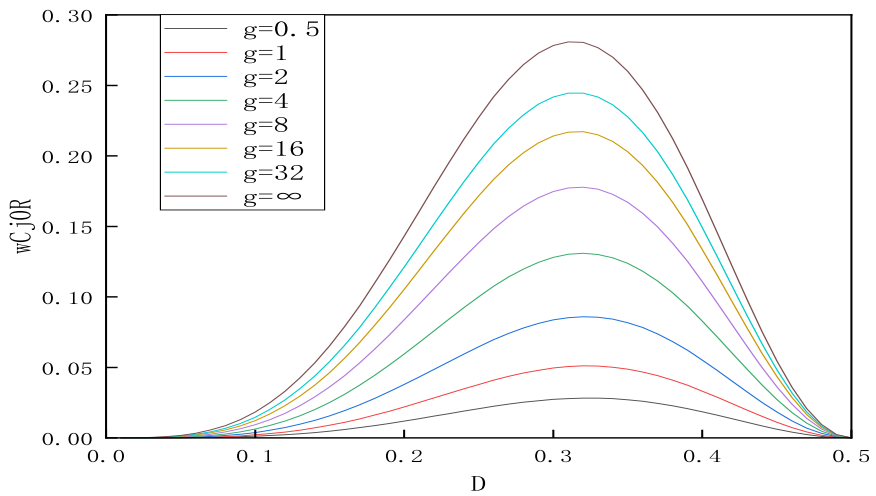

Fig. 3. $\omega C_{j 0} R$ versus $\mathrm{D}$ for selected values of $g$ at $V_{D D} / V_{b i}=14.29$, $n=2$.

The relationship between $I_{D D}$ and $I_{n}$ can be revealed

$$
I_{D D}=I_{n} \frac{\cos \Phi-\cos (x+\Phi)}{x}
$$

Where $x=2 n \pi D$, and ignoring power losses, input power equals output power,then

$$
I_{D D} V_{D D}=I_{n}^{2} R / 2
$$

combining (10) and (11), the amplitude of the output current can be expressed by $V_{D D}$ and $R$

$$
I_{n}=\frac{2 V_{D D}}{R} \frac{\cos \Phi-\cos (x+\Phi)}{x}
$$

And the amplitude of output voltage is

$$
V_{n}=R I_{n}=2 V_{D D} \frac{\cos \Phi-\cos (x+\Phi)}{x}
$$

The de input current is

$$
I_{D D}=\frac{I_{n}^{2} R}{2 V_{D D}}=\frac{2 V_{D D}}{R}\left(\frac{\cos \Phi-\cos (x+\Phi)}{x}\right)^{2}
$$

The output power can be expressed as

$$
P_{o}=\frac{I_{n}^{2} R}{2}=\frac{2 V_{D D}}{R V_{D D}}\left(\frac{\cos \Phi-\cos (x+\Phi)}{x}\right)^{2}
$$

\section{B. ZVDS Condition}

Introducing the ZVDS condition, that is

$$
I_{C}(2 \pi D)=0
$$

Substuiting (16) into (2)

$$
I_{D D}=I_{n} \sin (x+\Phi)
$$

Then uniting (10), the following expression can be obtained

$$
\begin{gathered}
\tan \Phi=\frac{\cos x+x \sin x-1}{\sin x-x \cos x} \\
\sin \Phi=\frac{\cos x+x \sin x-1}{Z} \\
\cos \Phi=\frac{\sin x-x \cos x}{Z}
\end{gathered}
$$

where

$$
Z=\sqrt{x^{2}+2-2 \cos x-2 x \sin x}
$$

Substituting (19) and (20) into (12)-(15), the following expressions can be obtained

$$
\begin{gathered}
I_{n}=\frac{2 V_{D D}}{R} \frac{1-\cos x}{Z} \\
V_{n}=2 V_{D D} \frac{1-\cos x}{Z} \\
I_{D D}=\frac{2 V_{D D}}{R} \frac{(1-\cos x)^{2}}{Z^{2}} \\
P_{o}=\frac{2 V_{D D}^{2}}{R} \frac{(1-\cos x)^{2}}{Z^{2}}
\end{gathered}
$$

\section{Component Values}

Refer to the assumptions 3), there is no resistance on $L_{R F C}$, which means

$$
\begin{aligned}
V_{D D} & =\frac{1}{2 \pi} \int_{0}^{2 \pi} V_{S} d \theta \\
& =\frac{1}{2 \pi} \int_{0}^{2 \pi D} 2 g V_{b i}\left(g+h(\theta)-\sqrt{g^{2}+2 g h(\theta)+1}\right) d \theta
\end{aligned}
$$

Then (26) can only be partially expanded as

$$
\begin{aligned}
\frac{V_{d d}}{V_{b i}}= & \frac{g}{\pi}\left(2 \pi D(g+1)+\frac{2 V_{D D}}{R} \frac{1-\cos x}{Z}(\sin (x+\Phi)\right. \\
& \left.-\sin \Phi+\frac{x^{2}(1-\cos x)}{2 Z}-x \cos \Phi\right) /\left(2 \omega C_{j 0} V_{b i} n^{2}\right) \\
& \left.-\int_{0}^{2 \pi D} \sqrt{g^{2}+2 g h(\theta)+1} d \theta\right)
\end{aligned}
$$

As can be seen in (27), $\omega C_{j 0} R$ is a function of $g$ and $D$. Fig.3 shows the variation relationship of $\omega C_{j 0} R$ with respect to $D$ when $V_{D D} / V_{b i}=14.29$ for selected g. The maximum value of $\mathrm{D}$ is 0.5 when $n=2$. Setting $g$ value from 0 to infinity, it can be seen that $\omega C_{j 0} R$ is always 0 when $g=0$ because there is no nonlinear capacitance in the shunt capacitance, which means $C_{j 0}=0$.when the value of $g$ grows, then the $\omega C_{j 0} R$ value gets bigger and bigger, and $\omega C_{j 0} R$ reaches the maximum value when $g=\infty$, which means that the shunt capaciancer only contains nonlinear capacitance. On the other hand, the $\omega C_{j 0} R$ value value increases from 0 to its peak as $\mathrm{D}$ increases from 0 to 0.324 and then decrease to 0 as $D=0.5$ for all selected $g$ values. The nth harmonic component $V_{S n}(\theta)$ of the switching voltage $V_{S}$ contains the output voltage $V_{o}(\theta)$ and the voltage $V_{L x}(\theta)$ across the reactance $L_{x}$

$$
\begin{aligned}
V_{S n}(\theta) & =V_{o}(\theta)+V_{L x}(\theta) \\
& =V_{o} \sin (n \theta+\Phi)+\frac{X}{R} V_{o} \cos (n \theta+\Phi) \\
& =V_{n} \sin \left(n \theta+\Phi_{1}\right)
\end{aligned}
$$

where $X=n \omega L_{x}$. The amplitude $V_{n}$ can be expressed as

$$
V_{n}=V_{o} \sqrt{1+\left(\frac{X}{R}\right)^{2}}
$$




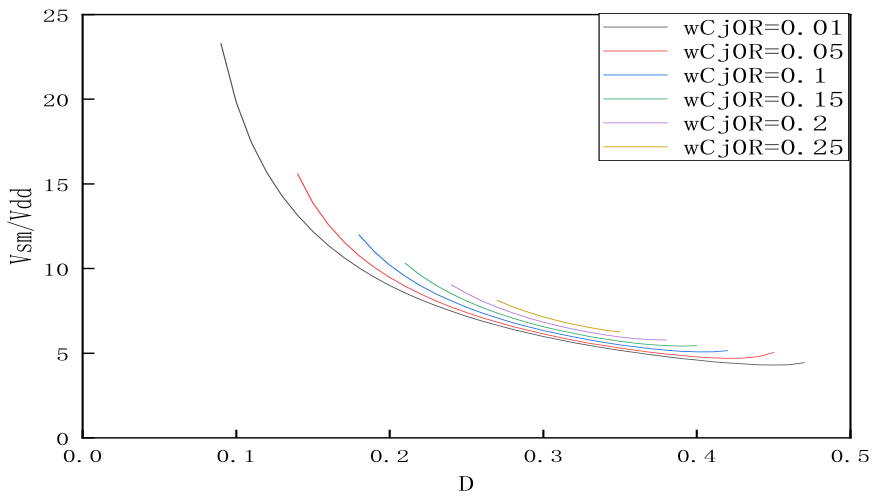

Fig. 4. $V_{S M}$ versus $D$ for selected $\omega C_{j 0} R$, at $V_{D D} / V_{b i}=14.29, n=2$, $Q=15$.

and the phase of $\Phi_{1}$ can be determined as

$$
\Phi_{1}=\Phi+\arctan \left(\frac{X}{R}\right)
$$

The nth order fourier expansion of the switching voltage $V_{S}$ is expressed as

$$
\frac{1}{\pi} \int_{0}^{2 \pi D} V_{S} \cos \left(n \theta+\Phi_{1}\right) d \theta=0
$$

Transforming this expression, then $\Phi_{1}$ is described as

$$
\tan \Phi_{1}=\frac{\int_{0}^{2 \pi D}\left(g+h(\theta)-\sqrt{g^{2}+2 g h(\theta)+1}\right) \cos n \theta d \theta}{\int_{0}^{2 \pi D}\left(g+h(\theta)-\sqrt{g^{2}+2 g h(\theta)+1}\right) \sin n \theta d \theta}
$$

Then $X$ is obtained

$$
X=R \tan \left(\Phi_{1}-\Phi\right)
$$

Once $X$ is given, the redundant inductance $L_{x}$ can be obtained

$$
L_{x}=R \tan \left(\Phi_{1}-\Phi\right) /(n \omega)
$$

The inductance $L$ can be derived

$$
L=\frac{R Q}{n \omega}
$$

and the series capacitance $C$ is given by

$$
C=\frac{1}{(n \omega)^{2}\left(L-L_{x}\right)}
$$

\section{Output Power Capability}

The switch peak current $I_{S M}$ is the sum of the dc supply current and the amplitude of the output current $I_{n}$

$$
\begin{aligned}
I_{S M} & =I_{D D}+I_{n} \\
& =\frac{2 V_{D D}}{R} \frac{1-\cos x}{Z}\left(1+\frac{1-\cos x}{Z}\right)
\end{aligned}
$$

The switch peak voltage $V_{S M}$ occurs when the shunt current $I_{C}$ is zero at $\theta=\theta_{1}$, i.e., $V_{S M}=V_{S\left(\theta_{1}\right)}$,where

$$
\theta_{1}=\frac{-\Phi+\arcsin ((1-\cos x) / Z)}{n}
$$

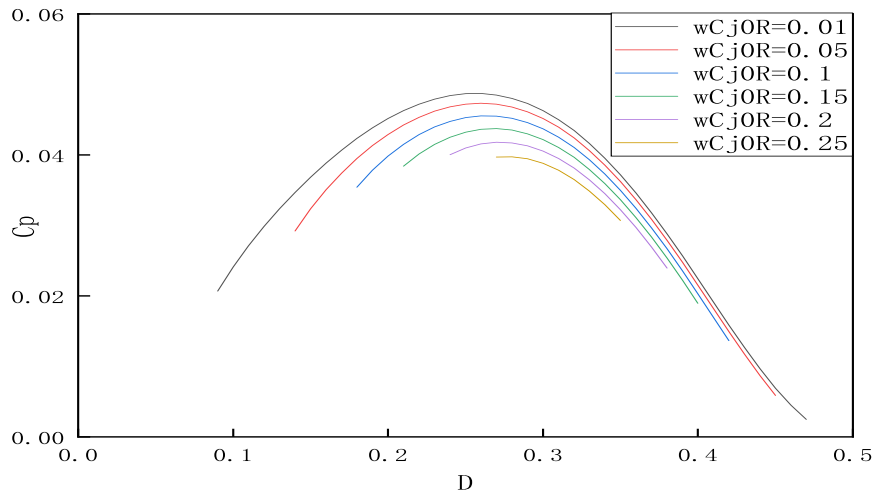

Fig. 5. $C_{p}$ versus $\omega C_{j 0} R$ for selected $D$ at $V_{D D} / V_{b i}=14.29, n=2$

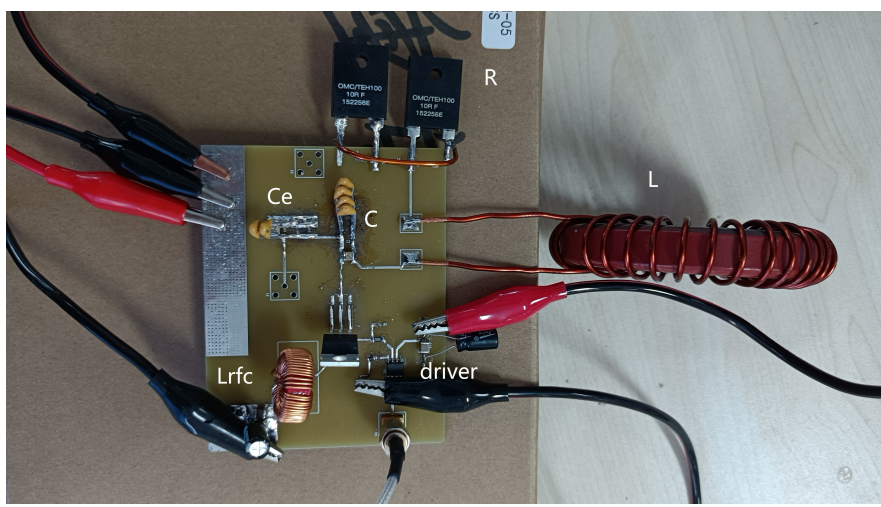

Fig. 6. photograph of the experimental frequency doubler

Fig.4 shows the relationship between the peak voltage $V_{S M}$ and $D$ for different values of $\omega C_{j 0} R$. The following conclusions can be obtained from the figure. First, the value of $\omega C_{j 0} R$ affects the value range of $D$ when the peak voltage $V_{S M}$ exists. The larger the value of $\omega C_{j 0} R$, the smaller the range of the values of $D$. Then, the peak voltage $V_{S M}$ shows a downward trend, and the downward trend becomes slower with the increase of $D$. Finally, the value of $V_{S M}$ is slightly higher if $\omega C_{j 0} R$ takes a larger value for the same $D$ value. The output power cappability $C_{p}$ is defined as

$$
C_{p}=\frac{I_{D D} V_{D D}}{I_{S M} V_{S M}}
$$

Fig.5 shows the relationship between $C_{p}$ and $D$ for selected $\omega C_{j 0} R$ at $V_{D D} / V_{b i}=14.29, n=2$. It can be seen that the selection of $\omega C_{j 0} R$ determines the range of $D$ in which $C_{p}$ exists. $C_{p}$ is slightly higher when the value of $\omega C_{j 0} R$ is smaller. And the value of $D$ at the peak of $C_{p}$ increases with the increment of $\omega C_{j 0} R$.

\section{Simulation And Experimental Results}

An example frequency doubler circuit is given to verify this theory and its disign specifications are $V_{D D}=10 \mathrm{~V}, R=$ $20 \Omega, D=0.25$ and $Q=15.25$ at operating frequency $f=1.5 \mathrm{MHz}$. In this experiment, The transistor IRF510 was selected, and the parameter value is $C_{j 0}=400 p F$, $V_{b i}=0.7 \mathrm{~V}$. The fabricated experimental frequency doubler is shown in Fig. 6. The value of $g$ can be determined as 
TABLE I

Theoretical And Simulated Peformance Class E Doubler, $Q=15.25$

\begin{tabular}{c|ccc|c}
\hline & $\begin{array}{c}\text { Theory } \\
\text { Values }\end{array}$ & $\begin{array}{c}\text { Simulation } \\
\text { Values }\end{array}$ & $\begin{array}{c}\text { Experiment } \\
\text { Values }\end{array}$ & $\begin{array}{c}\text { Difference } \\
\%\end{array}$ \\
\hline$R, \Omega$ & 20 & 20 & 20 & 0 \\
\hline$L, u H$ & 16.18 & 16.2 & 16.18 & 0 \\
\hline$C, p F$ & 188.69 & 188.3 & 196 & $3.8 \%$ \\
\hline$C_{e}, p F$ & 159.69 & 164 & 140 & $-12.3 \%$ \\
\hline$V_{S M}, V$ & 75.32 & 79.32 & 73 & $-2.9 \%$ \\
\hline$I_{S M}, A$ & 0.8254 & 0.875 & & \\
\hline$C_{p}$ & 0.0463 & 0.0438 & & \\
\hline$V_{o}(r m s), V$ & 7.6 & 7.65 & 7.33 & $-3.5 \%$ \\
\hline$P_{\text {in }}, W$ & 2.884 & 3.04 & 2.85 & $-1.2 \%$ \\
\hline$P_{\text {out }}, W$ & 2.884 & 2.924 & 2.686 & $-3.7 \%$ \\
\hline$\eta, \%$ & 100 & 96.18 & 94.2 & $-5.8 \%$ \\
\hline $\mathrm{D}$ & 0.25 & 0.25 & 0.25 & $0 \%$ \\
\hline
\end{tabular}

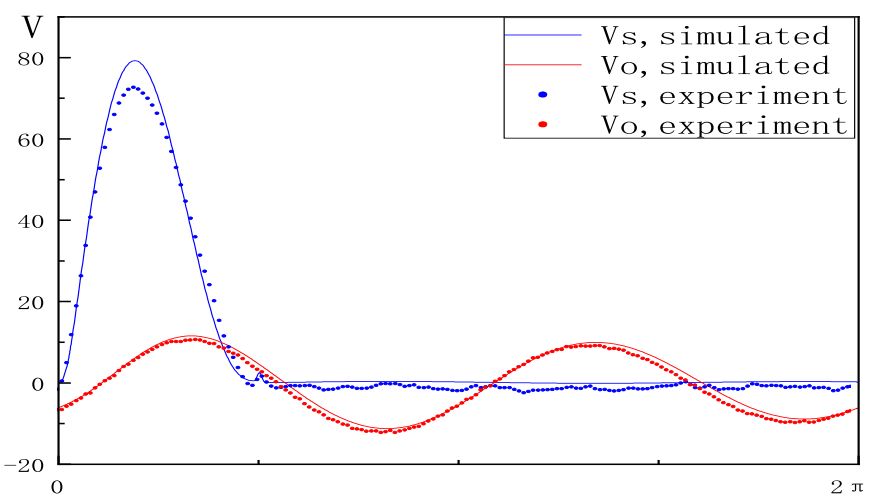

Fig. 7. The simulated and experimental waveform of $V_{S}$ and $V_{o}$

2.5 from (27). Then the values of all the circuit components can then be computed, the values of components in the circuit and some important circuit performance parameters was given in Table I. The series resonate inductor $\mathrm{L}$ was made in our lab and the value was measured by KEYSIGHT E5063A. As can be seen in Table I, the theoretical value, simulation value and experimental value of each component are very consistent. Fig.7 shows the drain-to-source voltage and output voltage waveforms obtained from ADS simulation and experimental results. It can be seen from Fig.7 and Table I that the theory, simulation and experimental results agrees well, which strongly proves the validity of the theory proposed in this paper.

\section{CONCLUSION}

An analysis, simulation and experiment of class E frequency multiplier with shunt nonlinear and linear capacitances at any duty ratio was presented. The effect of the duty ratio frequency multiplier is analyzed under the premise of the shunt capacitances composed of nonlinear and linear capacitances. A design equation for any harmonic output class E frequency multiplier was given. The theoretical results was validated by circuit simulation and experiment. The calculated and measured results were in great agreement.

\section{REFERENCES}

[1] N. Sokal and A. Sokal, "Class E-A new class of high-efficiency tuned single-ended switching power amplifiers," IEEE Journal of Solid-State Circuits, vol. 10, no. 3, pp. 168-176, 1975.

[2] F. Raab, "Idealized operation of the class E tuned power amplifier," in IEEE Transactions on Circuits and Systems, vol. 24, no. 12, pp. 725-735, December 1977, doi: 10.1109/TCS.1977.1084296.

[3] F. H. Raab, "Effects of circuit variations on the class E tuned power amplifier," in IEEE Journal of Solid-State Circuits, vol. 13, no. 2, pp. 239-247, April 1978, doi: 10.1109/JSSC.1978.1051026.

[4] F. H. Raab and N. O. Sokal, "Transistor power losses in the class E tuned power amplifier," in IEEE Journal of Solid-State Circuits, vol. 13, no. 6, pp. 912-914, Dec. 1978, doi: 10.1109/JSSC.1978.1052069.

[5] T. Kawahara and T. Suetsugu, "Class E frequency multiplier driving Class E amplifier," Proceedings of Power and Energy Systems in Converging Markets, 1997, pp. 617-621, doi: 10.1109/INTLEC.1997.646059.

[6] R. Zulinski and J. Steadman, "Performance evaluation of class E frequency multipliers," in IEEE Transactions on Circuits and Systems, vol. 33, no. 3, pp. 343-346, March 1986, doi: 10.1109/TCS.1986.1085903.

[7] R. Zulinski and J. Steadman, "Idealized Operation of Class E Frequency Multipliers," in IEEE Transactions on Circuits and Systems, vol. 33, no. 12, pp. 1209-1218, December 1986, doi: 10.1109/TCS.1986.1085880.

[8] M. Albulet, "Analysis and design of the Class E frequency multipliers with RF choke," in IEEE Transactions on Circuits and Systems I: Fundamental Theory and Applications, vol. 42, no. 2, pp. 95-104, Feb. 1995, doi: $10.1109 / 81.372849$

[9] M. Albulet and R. E. Zulinski, "Effect of switch duty ratio on the performance of class E amplifiers and frequency multipliers," in IEEE Transactions on Circuits and Systems I: Fundamental Theory and Applications, vol. 45, no. 4, pp. 325-335, April 1998, doi: 10.1109/81.669055.

[10] T. Suetsugu, "Novel operating condition of class E frequency multiplier which enables 50\% duty ratio," Proceedings of the 39th Midwest Symposium on Circuits and Systems, 1996, pp. 249-252 vol.1, doi: 10.1109/MWSCAS.1996.594118.

[11] M. Thian and V. Fusco, "Even Order Harmonic Series-L/Parallel-Tuned Class-E Frequency Multiplier," in IEEE Transactions on Circuits and Systems II: Express Briefs, vol. 54, no. 11, pp. 969-973, Nov. 2007, doi: 10.1109/TCSII.2007.903210.

[12] T. Suetsugu and M. K. Kazimierczuk, "Comparison of class-E amplifier with nonlinear and linear shunt capacitance," in IEEE Transactions on Circuits and Systems I: Fundamental Theory and Applications, vol. 50, no. 8, pp. 1089-1097, Aug. 2003, doi: 10.1109/TCSI.2003.815208.

[13] P. Alinikula, K. Choi and S. I. Long, "Design of Class E power amplifier with nonlinear parasitic output capacitance," in IEEE Transactions on Circuits and Systems II: Analog and Digital Signal Processing, vol. 46, no. 2, pp. 114-119, Feb. 1999, doi: 10.1109/82.752911.

[14] M. J. Chudobiak, "The use of parasitic nonlinear capacitors in class E amplifiers," in IEEE Transactions on Circuits and Systems I: Fundamental Theory and Applications, vol. 41, no. 12, pp. 941-944, Dec. 1994, doi: 10.1109/81.340867.

[15] D. K. Choi and S. I. Long, "The effect of transistor feedback capacitance in class-E power amplifiers," in IEEE Transactions on Circuits and Systems I: Fundamental Theory and Applications, vol. 50, no. 12, pp. 1556-1559, Dec. 2003, doi: 10.1109/TCSI.2003.819815.

[16] T. Suetsugu and M. K. Kazimierczuk, "Analysis and design of class E amplifier with shunt capacitance composed of nonlinear and linear capacitances," in IEEE Transactions on Circuits and Systems I: Regular Papers, vol. 51, no. 7, pp. 1261-1268, July 2004, doi: 10.1109/TCSI.2004.830695.

[17] A. Mediano, P. Molina-Gaudo and C. Bernal, "Design of Class E Amplifier With Nonlinear and Linear Shunt Capacitances for Any Duty Cycle," in IEEE Transactions on Microwave Theory and Techniques, vol. 55, no. 3, pp. 484-492, March 2007, doi: 10.1109/TMTT.2006.890512.

[18] M. Hayati, A. Lotfi, M. K. Kazimierczuk and H. Sekiya, "Analysis and Design of Class-E Power Amplifier With MOSFET Parasitic Linear and Nonlinear Capacitances at Any Duty Ratio," in IEEE Transactions on Power Electronics, vol. 28, no. 11, pp. 5222-5232, Nov. 2013, doi: 10.1109/TPEL.2013.2247633. 